\title{
Development of a PMU-Based Wind Power System Model via Modified Ward Reduction
}

This paper was downloaded from TechRxiv (https://www.techrxiv.org).

\section{LICENSE}

CC BY-NC-SA 4.0

SUBMISSION DATE / POSTED DATE

02-02-2021 / 03-02-2021

\section{CITATION}

McCann, Roy; Bowman, Douglas; Saadeh, Mahmood; Al-Sarray, Muthanna (2021): Development of a PMUBased Wind Power System Model via Modified Ward Reduction. TechRxiv. Preprint.

https://www.techrxiv.org/articles/preprint/Development_of_a_PMU-

Based_Wind_Power_System_Model_via_Modified_Ward_Reduction/13683928 


\title{
Development of a PMU-Based Wind Power System Model via Modified Ward Reduction
}

\author{
Muthanna Al-Sarray, Student Member, IEEE, Roy A. McCann, Senior Member, IEEE, Mahmood \\ Saadeh, Member, IEEE, and Douglas Bowman, Senior Member, IEEE
}

\begin{abstract}
Increasing amounts of wind generation in power systems creates challenges for transmission planners. Consequently, there is a need for improved models for optimizing the utilization of wind energy resources. This paper presents two main aims; a method for building small-scale models mimicking actual large systems and a new wind model that could be utilized for dynamic planning studies. The proposed method includes a Ward reduction technique with a specific procedure to extract models. After having derived a reduced equivalency, the new model is calibrated with phasor measurement units (PMUs) to adjust and then confirm the accuracy of benchmark models under various pre-fault and post-fault conditions. The method is applied to a large-scale power system with high penetration of wind generation. This application results in having a wind benchmark model that highlights the influences of large scale and remotely located wind generation under various dynamic conditions. The new model is evaluated through several validation steps. It is shown that the new wind model method provides very good accuracy and also accounts for breaker operations resulting from contingency events.
\end{abstract}

Index Terms--benchmark model, phasor measurement units, power flow, synchrophasor, Ward reduction method.

\section{INTRODUCTION}

$\mathrm{G}$ rowth of wind resource penetration in the United States and other countries due to clean environment policies, low incremental costs, and production tax credits (PTC) brings about issues impacting operational characteristics and dynamics of power systems ([1]-[3]). These levels of penetration increase both reliability and stability risks in power systems. Having power models accurately predict electric power systems for studying and analyzing the influences of wind generation helps planners and policy makers gain an overview of their systems and provides for optimization of power system operation when large amounts of wind generation is present. Small real-world models facilitate analysis of causes of line congestion, thermal limits, negative locational marginal pricing, wind curtailment, etc. Therefore, reduced models must predict realistic performance under various conditions with small errors. Performance errors during examination should meet requirements of the North American Electric Reliability Corporation (NERC) standards [4], [5].
Several methods are employed by researchers to build various small models for power analysis. These include Ward reduction technique, Kron reduction approach, Dimo method, and Zhukov method, after extracting behavior from large-scale networks [6]. The Ward reduction method is used widely by power systems researchers. This method partitions a large system into an internal part for detailed study and an external portion that accounts for the remainder of the system. The buses are divided into three types: (1) internal; (2) boundary; and (3) external. This process works through performing triangular reduction (Gaussian elimination) on the admittance matrix of networks. The internal system has all the details of the power system under analyzed while an external system may be ignored depending on the purposes of the study [7], [8]. For example, a Ward equivalent method in [9] was used to reduce the 68-bus New England system with constant PQ loads with benefits from PMUs data were gained through its use in analysis.

Both the standard and extended Ward equivalents in [10] were applied to the Netherlands transmission system to produce a model with a 65-bus test system by reducing the external system to boundary nodes. The results in [11] highlight the optimization of reactive power flow during the development of a new decomposition and coordination algorithm based on the Ward equivalent process. A modified load shedding algorithm combined with the Ward reduction method in [12] was used to evaluate the reliability of a regional power grid of Southern China that was reduced to 22 buses or $0.72 \%$ of the entire system. In [13], a reduction process was applied to the IEEE 118-bus system via an optimization Ward reduction approach to produce the 35-bus reduced model for optimal power flow purposes. From previous works, the Ward reduction technique was used to build small models simulating initial systems as this method keeps the studied area as an internal system with full details [7]. A modified Ward reduction process is developed in this work to produce models having full details of the studied area. The new model consists of all wind farms with small buses from the external system for achieving generation-demand balance.

The developed strategy in this work includes, in addition to

This research work is sponsored by the National Science Foundation (NSF) Industry/University Cooperative Research Center on Grid-connected Advanced Power Electronics Systems (GRAPES), GR-18-02 .

Muthanna Al-Sarray is with National Dispatch and Control Center, Ministry of Electricity, Baghdad, Iraq. He was with the Department of Electrical Engineering University of Arkansas, Fayetteville, AR 72701 USA (e-mail: maalsarr@uark.edu).

Roy A. McCann is with the Department of Electrical Engineering, University of Arkansas, Fayetteville, AR 72701 USA (e-mail: rmccann@uark.edu). Mahmood Saadeh is with Department of Electrical Engineering, Hashemite University, Az-Zarqa, Jordan (e-mail: saadeh_mahmood@yahoo.com). Douglas Bowman is with Southwest Power Pool, Little Rock, AR, 72223 USA (e-mail: dbowman@spp.org). 
a modified Ward equivalent technique in the model reduction processes, a validation procedure using actual operational system measurement data. Building models with high wind generation and calibrating the results of the model's simulation with PMUs measurements are a significant contribution of this paper. A new power system model in this work has been extracted from an actual system including numerous wind farms. The essential difference between this new model and those of previous research is in highlighting the response of breakers in transmission systems under variable generation and contingency (fault) events. The accuracy in the measurements of the new model reflects two points: The developed method builds reduced (simplified) models replicating large-scale power systems; and second, the new model in this paper gives planners an opportunity to study dynamic events in transmission systems with variable energy resources.

The paper is organized as follows: Section II introduces the developed Ward technique and the reduced model validating processes; Section III shows the validation results through various types of comparisons; Section IV provides a discussion of the result; and Section V provides conclusions.

\section{MODIFIED WARD REDUCTION METHOD}

The Modified Ward equivalent technique presented in this work is similar to the original Ward reduction method. However, it has new steps to select either buses or lines from an operational model and incorporates real-time measurement data. The proposed system is derived from an operational model simulated in PowerWorld environment that is used for application of the developed reduction method. The original operations model has conditions similar to those in the PMU data for accuracy aims. The studied network, referred to as Network6, is comprised of 15310 buses, 19931 lines, 2204 generators, and 69 areas with various voltage levels. Network6 includes large amounts of surplus wind generation capacity transported by transmission systems to demand buses in remote areas. The developed Ward reduced approach is divided into following two main procedures and is described in the following sections.

\section{A. Reduction Part}

The Ward reduction technique divides any studied system into two areas: (a) internal and (b) external. The buses are apportioned to internal (I), boundary (B), and external buses (E), as shown in Fig. 1. The bus admittance matrix (Y) for a large scale system can be written as in [8],

$$
Y=\left[\begin{array}{ccc}
Y_{E E} & Y_{E B} & 0 \\
Y_{B E} & Y_{B B}^{E}+Y_{B B}^{I} & Y_{B I} \\
0 & Y_{I B} & Y_{I I}
\end{array}\right],
$$

where $Y_{E E}$ and $Y_{I I}$ are the bus admittances of the respective external and internal systems; $Y_{E B}, Y_{B E}, Y_{I B}$, and $Y_{B I}$ are the bus admittances connecting the boundary buses with external and internal buses; $Y_{B B}^{E}$ and $Y_{B B}^{I}$ are the bus admittances for all lines between boundaries and external and internal buses, respectively. In the reduction method, the external system is equivalenced while the internal system is kept unchanged

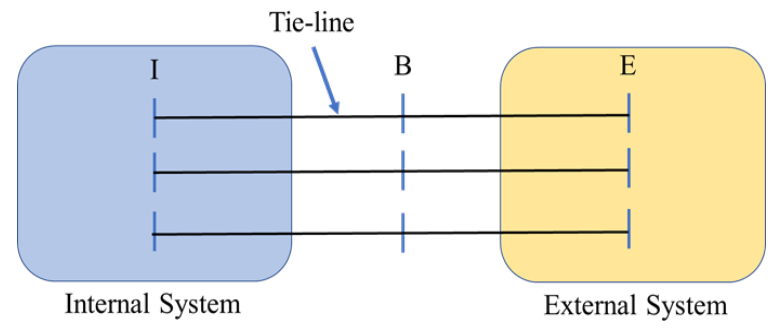

Fig. 1. Studied system before the Ward reduction method [8].

From the buses' perspective, the boundary and external buses are reduced to the following form,

$$
Y_{E B}^{E Q}=\left[\begin{array}{ll}
Y_{E E} & Y_{E B} \\
Y_{B E} & Y_{B B}^{E}
\end{array}\right]
$$

The lines connecting the boundary buses to the internal system are not influenced by the equivalencing process while lines connecting boundary buses to external system are reformed [8] as shown in Fig. 2. Equation (1) can be written in a different form if the voltage or current for both internal and external systems are considered [14];

$$
\begin{gathered}
I=Y V, \\
{\left[\begin{array}{l}
I_{I} \\
I_{E}
\end{array}\right]=\left[\begin{array}{ll}
Y_{I I} & Y_{I E} \\
Y_{E I} & Y_{E E}
\end{array}\right] \times\left[\begin{array}{c}
V_{I} \\
V_{E}
\end{array}\right],}
\end{gathered}
$$

where $I_{I}$ and $I_{E}$ are the injection currents at internal and external buses respectively and $V_{I}$ and $V_{E}$ are voltages at internal and external buses respectively. Eliminating the external system reformulates (4) into

$$
I_{I}=\left(Y_{I I}-Y_{I E} Y_{E E}^{-1} Y_{E I}\right) \times V_{I}+Y_{I E} Y_{E E}^{-1} I_{E}
$$

where the $Y_{I E} Y_{E E}{ }^{-1} Y_{E I}$ part leads to new equivalent branches, and branch terms in this paper means equivalent lines connecting boundary buses, while the $Y_{I E} Y_{E E}{ }^{-1} I_{E}$ part provides equivalent currents at boundary buses [14].

In this paper, internal and external systems are selected depending on positions of wind farms and load areas in Network6. This selection is required to classify the buses as internal, boundary, or external. The availability of buses in PMU data is also employed in the reduction process to avoid any lack of components that may result in inaccurate measurements in the simulation. The developed reduction technique in this work is achieved in following steps:

\section{1) Area Reduction}

Selecting geographical areas where wind farms are located is the first step. In addition to wind plant locations, the areas of load buses are also chosen to be added to the internal system for achieving the balance between generation and demand. Area2, which is a subsection of Network6 consisting of surplus generation, is selected as a generating area while areas in the east of Network6 are opted as load areas for the new model. Areas where a transmission system links the generation and load areas are considered to be within the internal system to complete the power paths. Boundary buses having either generating components or considerable loads are chosen. The first step is completed when the 


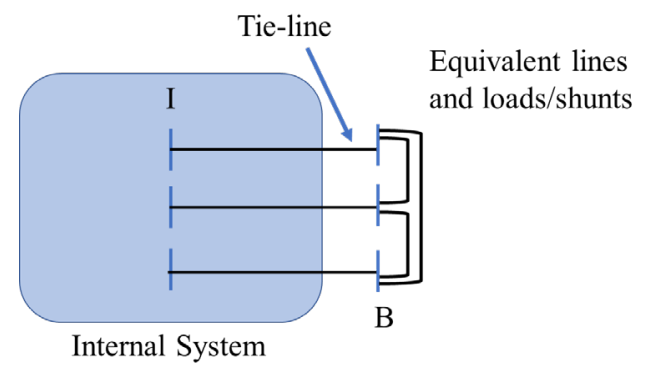

Fig.2. The internal system with the Ward equivalent connections [8].

internal system includes only the targeted generating, transmitting, and demand areas.

\section{2) Transmission Reduction}

The second step in the developed reduction method is selecting the main transmission levels. For this research, these are $345 \mathrm{kV}$ and $138 \mathrm{kV}$ for the modified system. The chosen transmission levels represent essential paths to transfer generating power from wind stations and other generating plants to load areas. Other transmission levels, such as $500 \mathrm{kV}$, $230 \mathrm{kV}$, and $161 \mathrm{kV}$ infrastructures are existent in the internal system as they provide paths for transferring power without overloading $345 \mathrm{kV}$ and $138 \mathrm{kV}$ lines. Most lines in the internal system are similar to lines in the original system except some new branches that connect boundary buses together from the Ward equivalent method ([8],[9],[15]) are not present in the initial system. By end of this step, the internal system has a transmission system with a reduced number of lines adequate to transfer power as in the original system.

\section{3) Substation Reduction}

The third step of the reduction method is eliminating undesired buses in the internal system. Criterion for selection of buses for the new system are wind farms, connecting buses, off-line status, and demand points. For explanation of these criteria, most of generating plants are either wind farms or other types of power plants in Area2. They are opted to be within the new system to produce the power required for power flow comparable to the original model. Moreover, the connecting buses are kept in the internal system because they are parts of power routes between generating and load buses. Maintaining these buses gives the small model more realistic conditions. The other selection criterion is the status of buses and lines in the original operations model provided by company Network6. Some of buses in the studied areas are eliminated in the new model because the lines connecting them with other buses are out of service. The last criterion is demand buses, especially with high amount of load, which are chosen in the new model to fulfill the cycle between the generation and demand. At this point in the process, the internal system has almost all its main components to complete a normal simulation comparable to the initial model.

\section{4) Internal Reduction}

Generally, substations comprise of different buses at various voltage levels connecting generators, transformers, shunt capacitors or reactor depending on the type of substations. The final step in this work, internal reduction, focuses on components of substations. Internal reduction means eliminating or shifting some electrical components in substations to different locations in the internal system. A criterion is utilized in this step to decrease the complexity of the new model. For instance, an amount of load $<10$ MVA and a small shunt capacitor or reactor is ignored due to low impacts $t$ that they have on the model performance. Also, generators or loads at low voltage levels are consolidated with higher voltage buses, especially if the maximum values of generators or demand points are small compared with the capacity of lines or transformers linking them to substations. Therefore, moving those components reduces the size of substations and the internal system. Moreover, shut admittances in the model are converted to loads for simplification as in (5).

Some substations components at low voltage levels, especially transformers connecting generators and loads to main buses, are kept in the new model to give planners and researchers the ability to analyze transformer behavior during studies. After this step, a 457-bus simplified wind equivalent system, WTS457, consisting of 457 buses, 98 generators, 786 lines, and 15 areas is considered complete. WTS457 has a transmission system with various voltage levels consisting of various substations with a high penetration of wind generation for mimicking Network6 with a low level of complexity.

\section{B. Validation Part}

After building the small wind model, the validation process begins. Power flow in the WTS457 is checked in order to test the accuracy of its measurements compared with measurements of the initial system for improved efficiency of operations and/or ensure power system security and stability. The WTS457 model is solved in PowerWorld Simulator 20 and a fault is applied in the WTS457 network. This results in a $345 \mathrm{kV}$ line trip comparable to an event in both the operational file and PMU data provided by Network6 and Area2 companies, respectively. The validation approach applied to the measurements of WTS457 model simulation consists of two main inspections; PMU measurements data comparison and Network6 model files comparison.

\section{1) PMU Measurements Data Comparison}

NERC advises utilities to validate small operation and planning models using synchrophasor data because the exact matching for power flows between original models and small models is not expected after reduction [16]. NERC Model Validation Working Group (MVWG) recommends that power flow errors for new models be within $+/-10 \%$ of measured values [17]. Therefore, this test is necessary for obtaining the error rate.

Synchrophasor technology supplies accurate standardized measurements of power system variables such as voltage, current, phase angles, and frequency at different locations in power systems via the common angle reference defined by a universal time signal in the Global Position System (GPS) framework [18]. In this paper, actual synchrophasor data is provided by the Area 2 company since the wind farms are mainly in the Network6. This data represents recorded synchrophasor measurements at 265 terminal IDs for a 
particular time duration and date. Synchrophasors provide time-stamped data with sampling rates of 30 frames per second. Each frame is composed of different variables such as a time stamp, terminal ID number, voltage and current magnitudes, voltage and current angles, frequency, and rate of change of frequency (ROCOF) [16]. The PMUs data included an actual fault that occurred in Network6.

Three PMUs data variables, voltage angles, active power, and reactive power are selected as main variables for validation of WTS457. However, direct comparison of voltage angles from PMUs and the simulated voltage angles in the new model must be aligned due to different references ([16],[18]). Some PMU measurements revealed a difference between the measured frequency of entire system of Area2, which its offnominal frequency is about $60.004 \mathrm{~Hz}$, and the reference frequency generated internally, which is $60 \mathrm{~Hz}$. This phenomenon causes increased phase angles and results in a wrapping effect, rotating the voltage of synchrophasors an additional cycle every $\sim 25$ seconds $(\sim 1 / 0.004$ s) [16]. This issue was treated in this work as in [16] to achieve a comparison without any potential errors.

The error in the voltage angle comparison is obtained by directly subtracting the voltage angles of benchmark model with the voltage angles of PMUs as

$$
\begin{gathered}
\delta_{j k}=\delta_{j}-\delta_{k}, \\
\delta_{j k}^{\text {error }}=\delta_{j k}{ }^{\text {Model }}-\delta_{j k}{ }^{\text {PMU }}
\end{gathered}
$$

where $\delta_{j}$ is voltage angle at bus $j, \delta_{k}$ is the voltage angle at bus $k, \delta_{j k}$ is the power angle between the buses $j$ and $k . \delta_{j k}{ }^{e r r o r}$ is the error resulting from a direct subtraction of voltage phase angles of WTS457 and PMUs measurements. $\delta_{j k}{ }^{\text {Model }}$ and $\delta_{j k}{ }^{P M U}$ is the phase of the WTS457 and PMUs, respectively.

The other calculations to complete the PMU comparison are made by taking into account the differences between active or reactive power flow, and the weight of the line that carries those powers. The weight of line, $w_{i}$, is a ratio of power passing through line $i$ to the net of powers passing through the lines of WTS457.

$$
w_{i}=\frac{P_{i}}{\sum_{i=1}^{n} P_{i}}
$$

where $w_{i}$ is the weight of line $i, P_{i}$ is the power passing through line $i$, and $\mathrm{n}$ is the number of lines in the benchmark model. The accuracy of the comparisons is obtained similar to [16] by calculating the relative error, $\varepsilon$

$$
\varepsilon=\frac{\left[\frac{1}{n} \sum_{i=1}^{n}\left(X_{i p m u}-X_{i \text { benchmak }}\right)^{2} w_{h}\right]^{\frac{1}{2}}}{\left[\frac{1}{n} \sum_{i=1}^{n} X_{i \mathrm{pmu}}{ }^{2}\right]^{\frac{1}{2}}}
$$

where $X_{i \text { pmu }}$ represents a power measured by PMU for line $I$ and $X_{i \text { benchmak }}$ is a power measured in the new model simulation for line $i$. Relative error is utilized to derive a measure of confidence $\sigma$ as given by

$$
\sigma=100 \%(1-\varepsilon) .
$$

For analysis purposes, the error percentage, $\epsilon_{i}$, for line $i$ is calculated as

$$
\epsilon_{i}=\left|\frac{X_{\text {ibenchmak }}-X_{\text {ipmu }}}{X_{\text {ipmu }}}\right| \times 100 \%
$$

and the weighted error, $W \epsilon_{i}$, is obtained as

$$
W \epsilon_{i}=\epsilon_{i} \times w_{i}
$$

This examination is deemed to be a quantities check as it focuses on the values of the variables.

\section{2) Network6 Model Files Comparison}

The second inspection in this work examines the direction of power flow in WTS457 compared with the initial model files. Different buses are selected in this comparison depending on location to emphasize accuracy of the reduced model. This comparison checks and validates both quantitative and directional values of power flow.

All previous comparisons applied on WTS457 are for a fault event under different conditions. The developed Ward reduction approach with its parts for validations is shown in Fig. 3. WTS457 is illustrated in Fig. 4 where the main transmission voltage systems (500kV and $345 \mathrm{kV})$ are highlighted. In Fig. 4, bold horizontal lines are buses, and thin lines are lines linking those buses. Circles with wind shapes represent wind farms connected either directly to ultra-high voltage levels or through step-up transformers. Circles with rotor ("dog bone") shapes are conventional generators, and bold vertical lines are $500 \mathrm{kV}$ transmission lines. Also, Fig. 5 shows wind farms included in Area2 with main lines transferring power to the rest of WTS457.

\section{RESULTS}

WTS457 covers major aspects of the targeted area in the Network6, such as wind generations, load points, and transmission levels, by having the same parameters as the initial system components with slight modifications. Some differences in the WTS457 parameters are either from the reduction technique or from modifications and are set to run the system normally. However, those variations should be acceptable to make model simulation measurements within the NERC standards and the MVWG requirements [17].

For evaluation purposes, a contingency event is applied to WTS457 to replicate an actual event that occurred in Area2 of Network6. In the initial model, Line 58-67 $(345 \mathrm{kV})$ was opened and this event was recorded by the PMUs. The same line in WTS457 experienced a trip condition and load flow around this line is analyzed due to being located near several wind farms. This test emphasizes both wind farms and system performance during the dynamic disturbance condition.

The model assessment is accomplished by analyzing results from 16 of the $345 \mathrm{kV}$ lines in the WTS457 model and comparing their power flow measurements with direct PMU measurements. These are tabulated in Fig. 5. The selection of these particular lines to validate the model is due to availability of direct load flow levels from the available PMU data.

The first variable inspected in this work are the voltage angles at buses to show the impacts of voltage angle variations on power flow and are shown in Tables I and II. The first column represents the number of the line, the second and third 


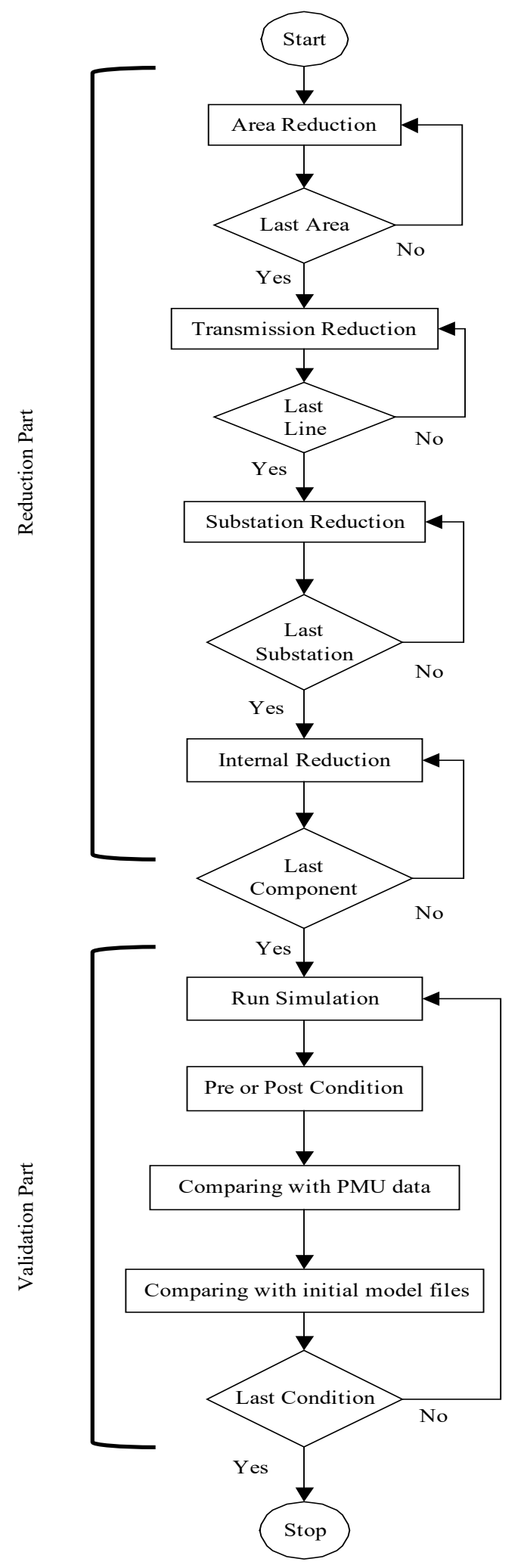

Fig. 3. Flow chart of modeling and validating part of the modified Ward Reduction Technique.

columns show voltage angle values for WTS457 and the PMU measurements for the same lines. The fourth column is the direct errors (6). In Tables I and II, the direct errors for voltage angles, computed in (7), in both conditions are within

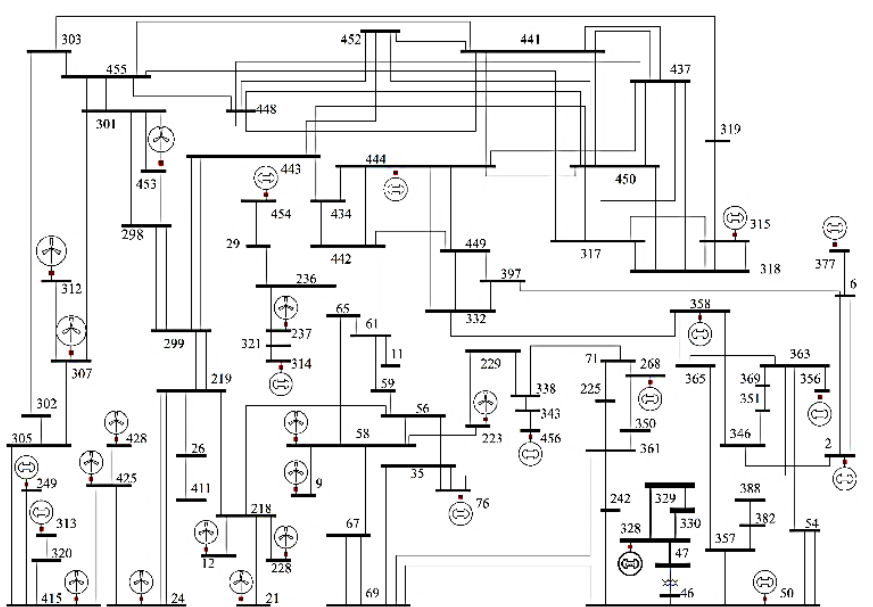

Fig. 4. One-line Diagram of $500 \mathrm{kV}$ and $345 \mathrm{kV}$ transmission system in WTS457.

$\pm 2.51^{\circ}$. For example, line $29-236345 \mathrm{kV}$ has an error of $2.51^{\circ}$ due to the change in power flow amount compared with PMU measurements. This variation in load flow comes from power production changes of several generators due to the change of demand in the reduced systems. The error in the phase angle of line $29-236345 \mathrm{kV}$ is decreased to $0.4^{\circ}$ due to altering the load flow after opening line $57-67345 \mathrm{kV}$. However, the outaged line, $57-67345 \mathrm{kV}$, shows a high error in phase angle value, which is $3.19^{\circ}$, because of the change of power flow paths after the fault.

In Tables III and IV, the comparisons are calculated for preand post-event conditions by taking into account the absolute values of active power in each line. In these tables, the second and third columns are active power values of WTS457 and the PMUs measurements for the same lines. The fourth and the fifth columns are the direct error and error percentages $\left(\epsilon_{i}\right)$ and the sixth and seventh columns are the line weights for active power $(W i)$ and weight error $\left(W \epsilon_{i}\right)$. In Table III, the errors between WTS457 and the measurements of PMU data are within \pm 50 MW. These errors are due to eliminating generators and load nodes in the external network. For instance, the line 56-35 $345 \mathrm{kV}$ has a large power error of $-49.16 \mathrm{MW}$.

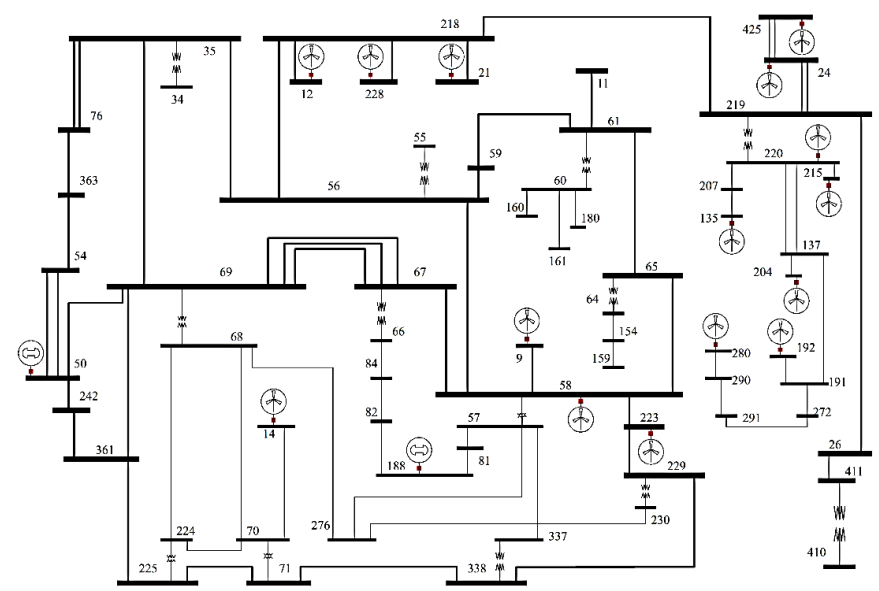

Fig. 5. One-line diagram of the wind farms area, Area2, with $345 \mathrm{kV}, 230 \mathrm{kV}$ and $138 \mathrm{kV}$ transmission systems of WTS457. 
The cause of this error is Bus $12345 \mathrm{kV}$, a wind farm, connected to bus Bus $218345 \mathrm{kV}$, that increases its wind generation compared with its generation in the initial system. Bus12 $345 \mathrm{kV}$ produced $152.02 \mathrm{MW}$ in the original system while it was increased to $189.8 \mathrm{MW}$ in simulation of WTS457 due to changing demand at load nodes and the elimination of some generators. These variations affected the power flow in lines. Therefore, lines $56-218345 \mathrm{kV}$ and $56-35345 \mathrm{kV}$ experienced this change in power flow, as shown in table III. Likewise, Table IV, representing the comparison of active power for the post-event condition, shows the same range of power errors between active power of the reduced model and the initial model. Using the calculations from (3) to (5), the accuracy of the quantities of active power is obtained for both conditions, $99.12 \%$ and $98.85 \%$, respectively.

The third variable checked is reactive power in the test area. Tables V and VI exhibit the differences in reactive power measurements for both WTS457 and PMUs measurements for

TABLE I

Voltage Angle Pre-Fault (DEG)

\begin{tabular}{lrrr}
\hline \hline Line & 457WS & PMU & \multicolumn{1}{c}{ Error } \\
\hline $219-26$ & 3.54 & 4.03 & -0.49 \\
$219-24$ & -2.04 & -2.01 & -0.03 \\
$219-218$ & -0.65 & -0.82 & 0.17 \\
$56-218$ & -8.24 & -6.56 & -1.68 \\
$56-58$ & 1.02 & 1.22 & -0.20 \\
$56-35$ & -0.43 & 1.67 & -2.10 \\
$61-59$ & 3.20 & 3.14 & 0.06 \\
$61-65$ & 2.07 & 2.31 & -0.24 \\
$236-65$ & 0.68 & 0.31 & 0.37 \\
$58-65$ & -2.54 & -2.29 & -0.25 \\
$58-223$ & 0.28 & 0.43 & -0.15 \\
$58-67$ & 2.85 & 2.44 & 0.41 \\
$35-69$ & 4.51 & 3.88 & 0.63 \\
$69-67$ & -0.21 & -1.89 & 1.68 \\
$29-236$ & 0.12 & -2.39 & 2.51 \\
$223-229$ & 1.23 & 1.98 & -0.75 \\
\hline \hline
\end{tabular}

TABLE II

Voltage Angle Post-Fault (DEG)

\begin{tabular}{lcrr}
\hline \hline Line & 457WS & \multicolumn{1}{c}{ PMU } & \multicolumn{1}{c}{ Error } \\
\hline $219-26$ & 3.53 & 3.90 & -0.37 \\
$219-24$ & -2.04 & -2.16 & 0.12 \\
$219-218$ & -0.70 & -0.86 & 0.16 \\
$56-218$ & -8.18 & -6.23 & -1.95 \\
$56-58$ & 0.47 & 0.81 & -0.34 \\
$56-35$ & 0.23 & -0.29 & 0.52 \\
$61-59$ & 3.10 & 2.54 & 0.26 \\
$61-65$ & 1.89 & 1.63 & 0.26 \\
$236-65$ & 0.64 & 0.26 & 0.38 \\
$58-65$ & -2.06 & -1.89 & -0.17 \\
$58-223$ & 0.54 & 0.66 & -0.12 \\
$58-67$ & 6.26 & 2.44 & 3.82 \\
$35-69$ & 5.93 & 5.44 & 0.49 \\
$69-67$ & 0.57 & 0.85 & -0.28 \\
$29-236$ & 0.06 & -0.34 & 0.40 \\
$223-229$ & 1.50 & 1.35 & 0.15 \\
\hline \hline
\end{tabular}

pre- and post-fault conditions, respectively. Similar to previous tables, Table V and VI have the same information except the second and third columns have the values of reactive power for both conditions. The divergence in error values of reactive power for both models is noticeable because the reactive power error has wide range of values, within \pm 100 MVAr. Those values of error between the both measurements are results of reconfiguration of the reduced model with removing the external network and relinking the boundary buses together ([9], [15]). Therefore, WTS457 does not have the same amount of reactive power produced by high voltage transmission lines because a number of lines are removed that helped the initial system to have reactive power flow without relying on generator supply. The accuracy of the quantities of reactive power is obtained for both conditions depending on (8) to (12). Compared with accuracy of active power, the accuracy values have low values, which are were $92.50 \%$ and $92.72 \%$ for preand post-failure conditions, respectively.

TABLE III

ACtive Power Pre-Fault (MW)

\begin{tabular}{|c|c|c|c|c|c|c|}
\hline Line & $457 \mathrm{WS}$ & PMU & Error & $\epsilon_{i}$ & $W i$ (p.u.) & $W \epsilon_{i}$ \\
\hline $219-26$ & 142.30 & 153.67 & 11.37 & 7.40 & 0.0024 & 0.017 \\
\hline $219-24$ & 73.80 & 84.79 & -10.99 & 12.96 & 0.0012 & 0.016 \\
\hline $219-218$ & 44.90 & 56.50 & -11.60 & 20.53 & 0.0007 & 0.015 \\
\hline $\begin{array}{lll}56 & -218\end{array}$ & 399.30 & 353.59 & 45.71 & 12.93 & 0.0066 & 0.085 \\
\hline $\begin{array}{lll}56 & -58\end{array}$ & 229.80 & 228.60 & 1.20 & 0.52 & 0.0038 & 0.002 \\
\hline $\begin{array}{ll}56 & -35\end{array}$ & 57.50 & 106.66 & -49.16 & 46.09 & 0.0010 & 0.044 \\
\hline $\begin{array}{ll}61 & -59\end{array}$ & 182.10 & 174.90 & 7.20 & 4.12 & 0.0030 & 0.012 \\
\hline $61-65$ & 167.20 & 184.23 & -17.03 & 9.24 & 0.0028 & 0.026 \\
\hline $236-65$ & 118.60 & 73.22 & 45.38 & 61.98 & 0.0020 & 0.122 \\
\hline $58-65$ & 156.90 & 133.01 & 23.89 & 17.96 & 0.0026 & 0.047 \\
\hline $58-223$ & 42.70 & 46.17 & -3.47 & 7.52 & 0.0007 & 0.005 \\
\hline $58-67$ & 285.40 & 237.50 & 20.17 & 20.17 & 0.0047 & 0.095 \\
\hline $35-69$ & 230.30 & 211.87 & 18.43 & 8.70 & 0.0083 & 0.033 \\
\hline $69-67$ & 7.70 & 1.39 & 6.32 & 455.96 & 0.0001 & 0.058 \\
\hline $29-236$ & 19.60 & 30.33 & -10.73 & 35.38 & 0.0003 & 0.011 \\
\hline $223-229$ & 212.10 & 218.71 & -6.61 & 3.02 & 0.0035 & 0.011 \\
\hline
\end{tabular}

The last check is verifying the net active and reactive power at boundary buses that should be equal to zero. In other words, the injected power to bus $k$ should be equal to the withdrawn power from bus $k$. For instance, the injected power at Bus 315 $345 \mathrm{kV}$ is equal to the withdrawn power and the net of two powers is zero. Bus $315345 \mathrm{kV}$ was selected due to having a negative active load node representing the imported power to the studied area from the external system to keep generation and demand in WTS457 balanced. Keeping this type of node avoids divergence in power flow calculations. The same check was completed for different buses, such as Bus $329500 \mathrm{kV}$, Bus 6 $345 \mathrm{kV}$, and Bus $455345 \mathrm{kV}$, and the net of power flow in these buses is equal to zero. These buses are located far from the wind farms and the studied area and the error in these nodes is expected to be high. However, this check confirms that WTS457 meets NERC requirement that power flow errors of a new model should be within $+/-10 \%$ of measured values [17] 
TABLE IV

ACTIVE POWER Post-FAult (MW)

\begin{tabular}{crrrrrc}
\hline \hline Line & 457WS & \multicolumn{1}{c}{ PMU } & Error & \multicolumn{1}{c}{$\epsilon_{i}$} & $W i$ (p.u.) & $W \epsilon_{i}$ \\
\hline $219-26$ & 142.30 & 158.30 & 16.08 & 10.15 & 0.0024 & 0.024 \\
$219-24$ & 73.50 & 82.80 & 9.30 & 11.23 & 0.0012 & 0.014 \\
$219-218$ & 47.90 & 68.80 & 20.90 & 30.38 & 0.0008 & 0.024 \\
$56-218$ & 396.30 & 334.05 & -62.25 & 18.64 & 0.0066 & 0.122 \\
$56-58$ & 101.90 & 111.41 & 9.51 & 8.54 & 0.0017 & 0.014 \\
$56-35$ & 29.60 & 39.43 & 9.83 & 4.93 & 0.0005 & 0.012 \\
$61-59$ & 176.80 & 168.60 & -8.20 & 4.86 & 0.0029 & 0.014 \\
$61-65$ & 152.30 & 170.32 & 18.02 & 10.58 & 0.0025 & 0.027 \\
$236-65$ & 110.10 & 63.53 & -46.57 & 73.31 & 0.0018 & 0.133 \\
$58-65$ & 127.40 & 102.89 & -24.51 & 23.82 & 0.0021 & 0.050 \\
$58-223$ & 87.90 & 70.60 & -17.30 & 24.50 & 0.0015 & 0.036 \\
$58-67$ & 0.00 & 0.00 & 0.00 & 0.00 & 0.0000 & 0.000 \\
$35-69$ & 303.50 & 264.07 & -39.43 & 14.93 & 0.0050 & 0.075 \\
$69-67$ & 30.60 & 52.47 & 21.87 & 41.68 & 0.0005 & 0.021 \\
$29-236$ & 11.10 & 46.70 & 35.60 & 76.23 & 0.0002 & 0.014 \\
$223-229$ & 257.20 & 255.24 & -1.96 & 0.77 & 0.0043 & 0.003 \\
\hline \hline
\end{tabular}

TABLE V

ReACTIVE POWER Pre-FAult (MVAR)

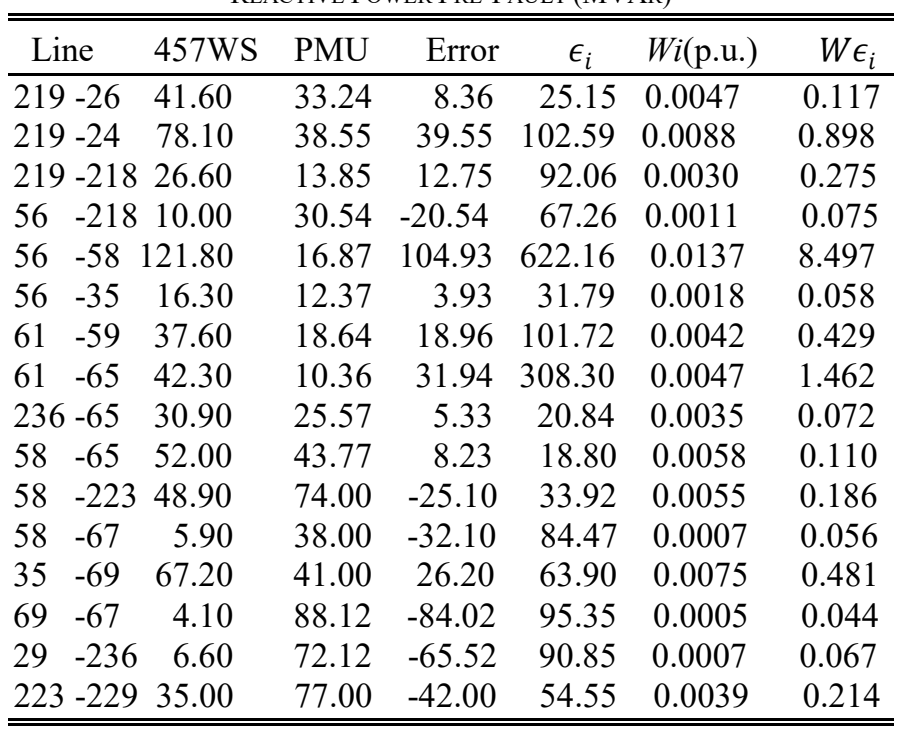

TABLE VI

ReACTIVE Power Post-Fault (MVAR)

\begin{tabular}{|c|c|c|c|c|c|c|c|}
\hline \multicolumn{2}{|c|}{ Line } & 457WS & PMU & Error & $\epsilon_{i}$ & $W i($ p.u. $)$ & $W \epsilon_{i}$ \\
\hline 219 & -26 & 41.60 & 33.00 & 8.60 & 26.06 & 0.0047 & 0.122 \\
\hline 219 & -24 & 78.10 & 38.00 & 40.10 & 105.53 & 0.0088 & 0.924 \\
\hline 219 & -218 & 26.80 & 59.34 & -32.54 & 54.84 & 0.0030 & 0.165 \\
\hline 56 & -218 & 11.40 & 34.85 & -23.45 & 67.29 & 0.0013 & 0.086 \\
\hline 56 & -58 & 119.30 & 12.23 & 107.07 & 875.47 & 0.0134 & 1.710 \\
\hline 56 & -35 & 20.40 & 17.20 & 3.20 & 18.60 & 0.0023 & 0.043 \\
\hline 61 & -59 & 37.10 & 15.87 & 21.23 & 133.77 & 0.0042 & 0.556 \\
\hline 61 & -65 & 42.30 & 38.68 & 3.62 & 9.36 & 0.0047 & 0.044 \\
\hline 236 & -65 & 30.00 & 20.95 & 9.05 & 43.20 & 0.0034 & 0.145 \\
\hline 58 & -65 & 50.00 & 43.21 & 6.79 & 15.71 & 0.0056 & 0.088 \\
\hline 58 & -223 & 53.90 & 93.25 & -39.35 & 42.20 & 0.0060 & 0.255 \\
\hline 58 & -67 & 0.00 & 0.00 & 0.00 & 0.00 & 0.0000 & 0.000 \\
\hline 35 & -69 & 55.40 & 49.88 & 5.52 & 11.07 & 0.0062 & 0.069 \\
\hline 69 & -67 & 1.90 & 46.55 & -44.65 & 95.92 & 0.0002 & 0.020 \\
\hline 29 & -236 & 7.40 & 74.30 & -66.90 & 90.04 & 0.0008 & 0.075 \\
\hline 223 & -229 & 34.40 & 79.56 & -45.16 & 56.76 & 0.0039 & 0.219 \\
\hline
\end{tabular}

The second verification of the results is to check the power flow directions in WTS457 as compared with the initial model file provided by company Network6. This check is conducted by contrasting the direction of power flow in both the Network6 file and the results of WTS457 simulation after running them in PowerWorld Simulator 20. The test includes two buses, one being is in the middle of targeted area, located near wind farms, and another being far from wind farms. The first bus is Bus58 $345 \mathrm{kV}$ located in the middle of the wind farms area and has some connected wind farms. The direction of active power flow for all lines and generators linked to this bus are the same for both the initial Network6 file and WTS457. For example, active power transfers from Bus58 $345 \mathrm{kV}$ to Bus $223345 \mathrm{kV}$ due to the voltage angle at Bus58 $345 \mathrm{kV}$, which is $-20.28^{\circ}$, is higher that the voltage angle at Bus223 $345 \mathrm{kV}$, which is $20.56^{\circ}$. The reactive power in this line flows in the opposite direction. To summarize, the reactive power flows from Bus223 345kV to Bus58 345kV due to the voltage magnitude at Bus223 345kV, which is 1.021 p.u., is higher than the voltage magnitude at Bus58 $345 \mathrm{kV}$, which is 1.017 p.u.

For an additional accuracy check, the direction of both active and reactive power flow is the same for WTS457, Network6 file, and PMUs measurements. Also, the comparison is applied too on Bus361 345kV, especially on line $69-361345 \mathrm{kV}$. The results are like previous example showing that all power flow of active and reactive power, for the initial files and the reduced system, have the same directions. All previous directional comparisons are tabulated in Table VII.

TABLE VII

POWER FLOW DIRECTION COMPARISON

\begin{tabular}{cccrcrc}
\hline \hline Line & \multicolumn{2}{c}{ 457WS } & \multicolumn{2}{c}{ Original Model } & \multicolumn{2}{c}{ PMU } \\
& MW & MVAr & MW & MVAr & MW & MVAr \\
\hline $58-223$ & 42.7 & -48.9 & 46.8 & -49.3 & 48.2 & -75.83 \\
$69-361$ & 37.5 & -91.8 & 0.7 & -85.7 & 3.0 & -88.92 \\
\hline \hline
\end{tabular}

\section{DISCUSSION}

The previous comparisons are made under steady state conditions. Therefore, an additional test is applied on WTS457 model to simulate its dynamic response during the fault disturbance. This final simulation is dependent on the PMUs measurements as these are compared them with the dynamic simulation of WTS457 in PowerWorld Simulator. Performances of phase angles, active, and reactive power calibrated with PMUs data are presented in Fig. 6-8. In these figures, the WTS457 model performance is similar to that of the original system by modeling the changes in load flow variables when the fault occurs at 0.5 seconds.

In Fig. 6, phase angles of the three lines show the same actions with small errors for both measurements for the WTS457 model and the PMUs data. For Fig. 7, active power for three lines experience the same changes after tripping Line 58-67 345kV. Reactive power in the three lines in Fig. 8 has some differences between measurements for the WTS457 model and the PMUs data. 


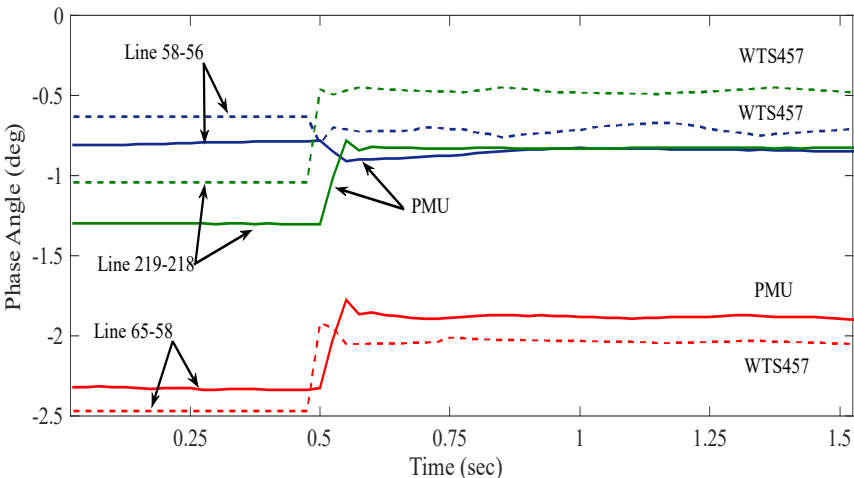

Fig. 6. Phase angles in three $345 \mathrm{kV}$ lines for both WTS457 and PMUs data after tripping Line $58-67345 \mathrm{kV}$.

Overall, the new model demonstrates most aspects of the actual system. The accuracy of the WTS457 model is within NERC requirements for validation of new models. Therefore, the WTS457 could be used for system planning.

\section{CONCLUSION}

The modeling method developed in this paper provides a process for researchers and planners to produce accurate models of large scale power systems with high accuracy.

The validation process was described to calibrate the model with PMUs measurement data. The validation steps of the WTS457 model showed small errors that met NERC requirements for model validation. WTS457 could be used when analyzing various operational circumstances where remotely located surplus wind capacity may exist.

\section{REFERENCES}

[1] Renewable Electricity Production Tax Credit (PTC). Energy Gov., Washington DC. [Online]. Available: https://energy.gov/savings/, Accessed on: Mar. 12, 2017.

[2] T. Beckford. (2017, May). Wind Turbines Provide $8 \%$ of U.S. Generating Capacity, more than Any Other Renewable Source. The U.S. Energy Information Administration (EIA), Washington DC. [Online]. Available: https://www.eia.gov/todayinenergy/detail.php?id=31032, Accessed on: Feb. 15, 2017.

[3] S. Q. Bu, W. Du, H. F. Wang, Z. Chen, L. Y. Xiao, and H. F. Li, "Probabilistic analysis of small-signal Stability of large-scale power systems as affected by penetration of wind generation," IEEE Trans. Power Syst., vol. 22, no. 2, pp. 762-770, May. 2012.

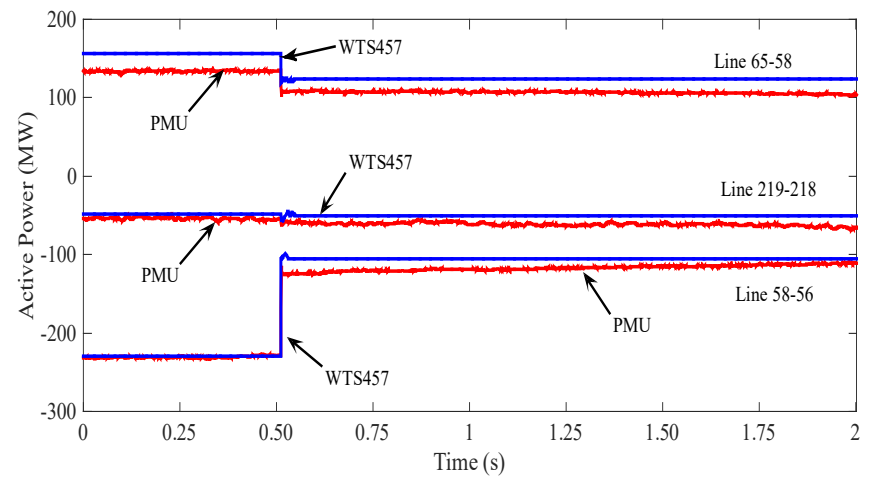

Fig. 7. Active power in three $345 \mathrm{kV}$ lines for both WTS457 and PMUs data after tripping Line 58-67 345kV.

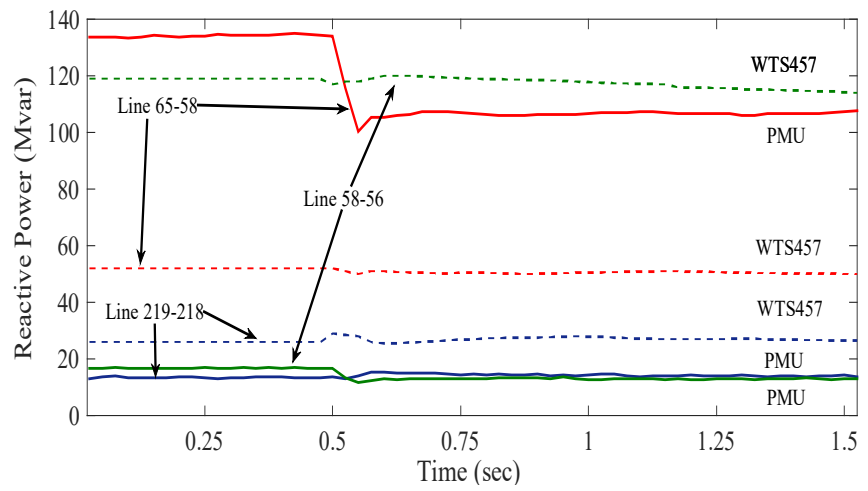

Fig. 8. Reactive power in three $345 \mathrm{kV}$ lines for both WTS457 and PMUs data after tripping Line $58-67345 \mathrm{kV}$.

[4] M. Garmroodi, D. J. Hill, G. Verbič, and J. Ma, "Impact of tie-line power on inter-area modes with increased penetration of wind power," IEEE Trans. Power Syst., vol. 31, no. 4, pp. 3051-3059, Jul. 2016.

[5] N. Nguyen, and J. Mitra, "Reliability of power system with high wind penetration under frequency stability constraint," IEEE Trans. Power Syst., vol. 33, Issue. 1, pp. 985-994, Jan. 2018.

[6] D. B. Payne and J. R. Stern, "Performance analysis of static network reduction methods commonly used in power systems," Nat. Power Syst. Conf. (NPSC), Guwahati, India, Dec. 18-20, 2014.

[7] H. Oh, "A New Network Reduction Methodology for Power System Planning Studies," IEEE Trans. Power Syst., vol. 9, no. 1, pp. 677-684, Feb. 1994.

[8] K. L. Lo, L.J. Peng, J. F. Macqueen, A. O. Ekwue, and N. H. Dandachi, "Extended Ward equivalent of external system for on-line security analysis," Int. Conf. on Advances in Power Syst. Control, Operation and Manage. (APSCOM-93), Hong Kong, Dec. 7-10, 1993.

[9] T. L. Baldwin, L. Mili, and A. G. Phadke, "Dynamic Ward equivalents for transient stability analysis," IEEE Trans. Power Syst., vol. 25, no. 2, pp. 59-67, May. 2010.

[10] R. A. M. van Amerongen, and H. P. van Meeteren, "A generalized Ward equivalent for security analysis," IEEE Trans. Power App. Syst., vol. PAS-101, no. 6, pp. 1519-1526, Jun. 1982.

[11] Z. W. Liu, and M. B. Liu, "Distributed reactive power optimization computing in multi-area power systems using Ward equivalent," Int. Conf. on Elect. and Control Eng., Wuhan, China, Jun. 25-27, 2010.

[12] H. Li, Z. Lu, Y. Qiao, and Z. Sun, "The fast reliability evaluation method in transmission grid expansion project based on power flow tracing and Ward equivalent method," Int. Conf. on Power Syst. Technol. (POWERCON 2014), Chengdu, China, Oct. 20-22, 2014.

[13] Y. Zhu, and D. Tylavsky, "An Optimization Based Network Reduction Method with Generator Placement," North American Power Symposium (NAPS2015), Charlotte, NC, Oct. 4-6, 2015.

[14] "Power System Equivalents," PowerWorld Corporation. Champaign, IL, 2008. [Online] Available: https://www.powerworld.com, Accessed on: Jan. 5, 2017.

[15] J. B. Ward, "Equivalent circuits for power-flow studies," Trans. of the American Inst. of Elect. Engineers, vol. 68, issue. 1, pp. 373-382, Jul. 1949.

[16] M. S. Saadeh, "Model Development and Validation for Wind Generation Transmission Systems," Ph.D. dissertation, Dept. Elect. Eng., Univ. of Arkansas, Fayetteville, AR, 2015.

[17] The NERC Model Validation Task Force of the Transmission Issues subcommittee. (2010, Dec.). White Paper on Power System Model Validation. North American Electric Reliability Corp., NJ. [Online]. Available:

http://www.nerc.com/docs/pc/mvwg/MV\%20White\%20Paper_Final.pdf Accessed on: Mar. 10, 2017.

[18] V. Venkatasubramanian, "Real-Time Strategies for Unwrapping of Synchrophasor Phase Angles," IEEE Trans. Power Syst., vol. 31, no. 6, pp. 5033-5041, Nov. 2016. 